\title{
Synthesis and post-synthetic modification of UiO-67 type metal-organic frameworks by mechanochemistry
}

\author{
Habiba Ali-Moussa ${ }^{\mathrm{a}, \mathrm{b}}$, Ricardo Navarro Amador ${ }^{\mathrm{b}}$, Jean Martinez ${ }^{\mathrm{a}}$, Frédéric Lamaty ${ }^{\mathrm{a}}$, Michaël Carboni ${ }^{\mathrm{b}}$, \\ Xavier Bantreil ${ }^{\mathrm{a}, *}$ \\ a Institut des Biomolécules Max Mousseron (IBMM), UMR 5247, CNRS, Université de Montpellier, ENSCM, Université de Montpellier Campus Triolet, Place Eugène Bataillon, \\ 34095 Montpellier cedex 5, France \\ b Institut de Chimie Séparative de Marcoule (ICSM), UMR 5257, CEA, CNRS, ENSCM, Université de Montpellier, Bat 426, BP 17171, 30207 Bagnols-sur-Cèze Cedex, France
}

Keywords:

Metal-organic framework

Functional porous material

Mechanochemistry

Ball-mill

Solvent-free reaction

\begin{abstract}
A B S T R A C T
Herein, we report the synthesis of zirconium metal-organic frameworks by a mechanochemical route. The frameworks are obtained by the coordination of a zirconium cluster with the biphenyldicarboxylic acid linker to obtain UiO-67 or with the iso-structural 2,2'-bipyridine-5,5'-dicarboxylic acid to access to a functional MOF (UiO-67-bpy). In a second mechanochemical reaction, it has been possible to coordinate by post-synthetic modification a copper salt on each bipyridine site (UiO-67-bpy-Cu). Both materials exhibit crystallinity and porosity (up to $750 \mathrm{~m}^{2} / \mathrm{g}$ ) showing the possibility to get access to elaborated materials in a cleaner way than the classical solvothermal route in DMF.
\end{abstract}

\section{Introduction}

Hybrid materials, combining switchable organic and robust inorganic moieties, are nowadays becoming more and more popular [1]. In this family, metal-organic frameworks (MOFs), which are nanoporous crystalline materials, are recognized for their versatile properties that go along with their high structural diversity [2]. They find numerous applications in drug delivery, [3] gas storage, [4] catalysis, [5] metal extraction, [6] etc. In particular, artificial photosynthesis has gained a lot of attention since MOF's structure can contain a photosensitizer and a catalytic center due to the presence of a coordination site on the structural linker [7]. Because of their high stability under light excitation or photocatalytic conditions, $\mathrm{Zr}$ and $\mathrm{Ti}$ MOFs are among the best candidates in this domain. Their syntheses often require harsh conditions and long reaction times, in toxic solvent such as DMF [8]. In addition, side-products arising from the metal source hydrolysis $\left(\mathrm{ZrCl}_{4}\right.$, $\mathrm{TiCl}_{4} \ldots$. ) are often obtained in these conditions [9]. To circumvent such issues, mechanochemistry by using ball-mills could become an excellent alternative to the classical solvothermal conditions. This method can also allow the synthesis of MOFs in a cleaner and larger production; two needs that are mandatory in order to

\footnotetext{
* Corresponding author.

E-mail address: xavier.bantreil@umontpellier.fr (X. Bantreil).
}

be introduced into the industry and a larger market. Indeed, working under solvent-free or solvent-less conditions, with an excellent agitation, allows to react efficiently highly concentrated mixtures, thus enhancing reaction rates. Moreover, since few quantities of solvent are used, substrate hydrolysis that occurs during MOF synthesis can be limited in ball-mills. Applications of ball-milling in various domains can be found in literature [10] but it is at an early stage of development when it is applied to the synthesis of MOFs [11]. In 2015, the group of Friščić demonstrated that it was highly convenient to synthesize UiO-66 or UiO-66- $\mathrm{NH}_{2}$, zirconium MOFs featuring terephtalic acid or 2-aminoterephtalic acid as organic ligands, respectively, using mechanochemistry as activation technique [12]. After less than $3 \mathrm{~h}$ of milling at $30 \mathrm{~Hz}$, MOFs with PXRD (powder X-ray diffraction) patterns and BET (Brunauer, Emmett and Teller) areas consistent with literature were obtained. Another interesting material from the same family of MOFs is the UiO-67, [13] which essentially is an analogue of the UiO-66 with bigger pores and surface area due to the increase of the length of the ligand 4,4'-biphenyldicarboxylic acid (BPDC) instead of the terephtalic acid but preserving the same morphology. In literature, synthesis of UiO-67 generally involves zirconium precursors such as $\mathrm{ZrCl}_{4}$ or $\mathrm{Zr}(\mathrm{On}-\mathrm{Pr})_{4}$, but recently, alternative routes involving the use of a pre-assembled clusters revealed promising [14].

Following our interest in the mechanochemistry involving organometallics species, [15-18] two Zr-based MOFs, the Uio-67 MOF 
and its bipyridine analogue (UiO-67-bpy) were synthesized by ball-milling in this work. This material was later modified by PSM (Post-Synthetic Modification) with $\mathrm{Cu}$ to obtain efficiently more complex MOF by mechanochemistry instead of using the classical solvothermal conditions.

\section{Results and discussion}

Benzoate and methacrylate clusters $1 \mathrm{Zr}_{6} \mathrm{O}_{4}(\mathrm{OH})_{4}\left(\mathrm{C}_{6} \mathrm{H}_{5} \mathrm{CO}_{2}\right)_{12}$ and $2 \mathrm{Zr}_{4} \mathrm{O}_{2}\left(\mathrm{C}_{2} \mathrm{H}_{3} \mathrm{CO}_{2}\right)_{12}$ were prepared as previously described in literature. Zirconium sources were then ball-milled, using a Retsch

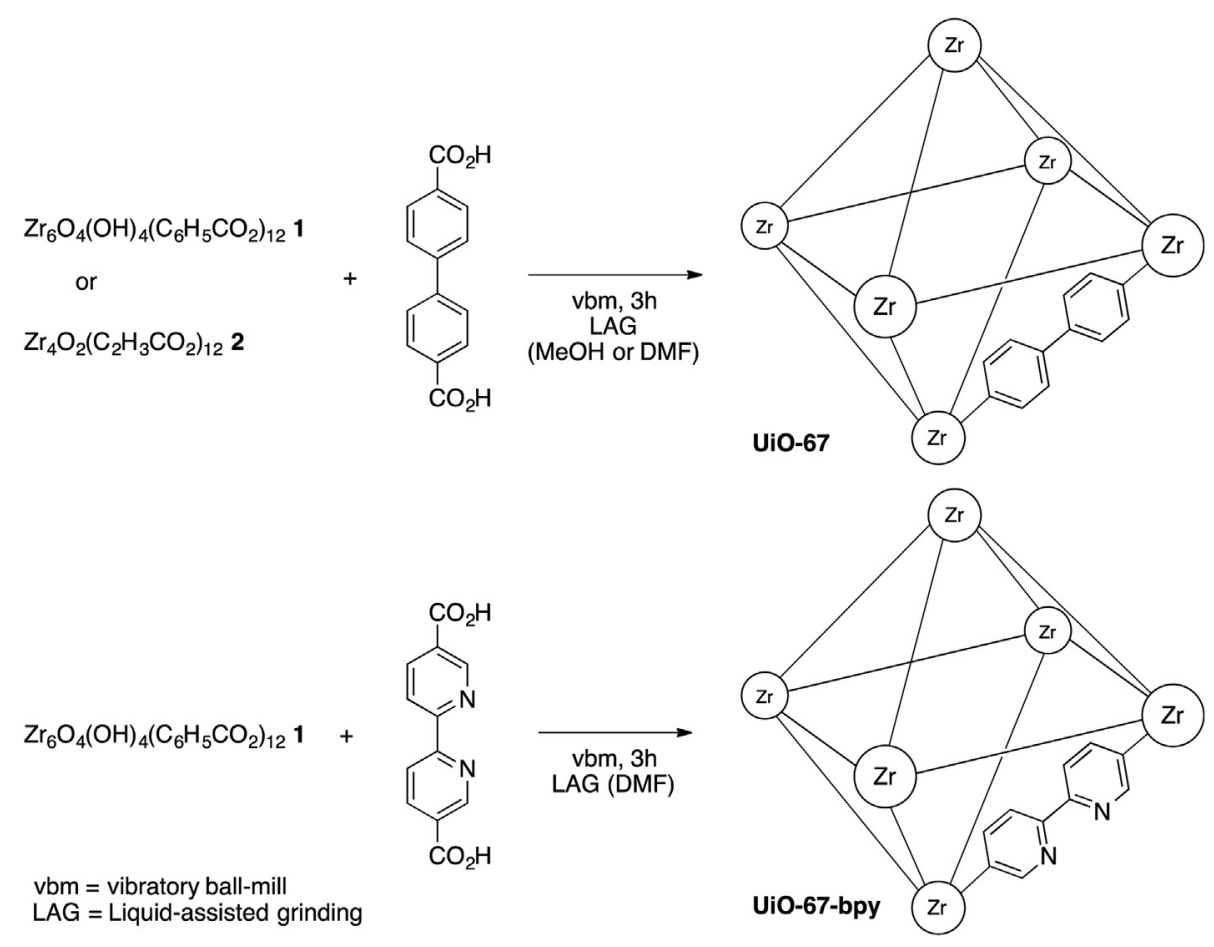

Scheme 1. Mechanochemical synthesis of UiO-67 and UiO-67-bpy.
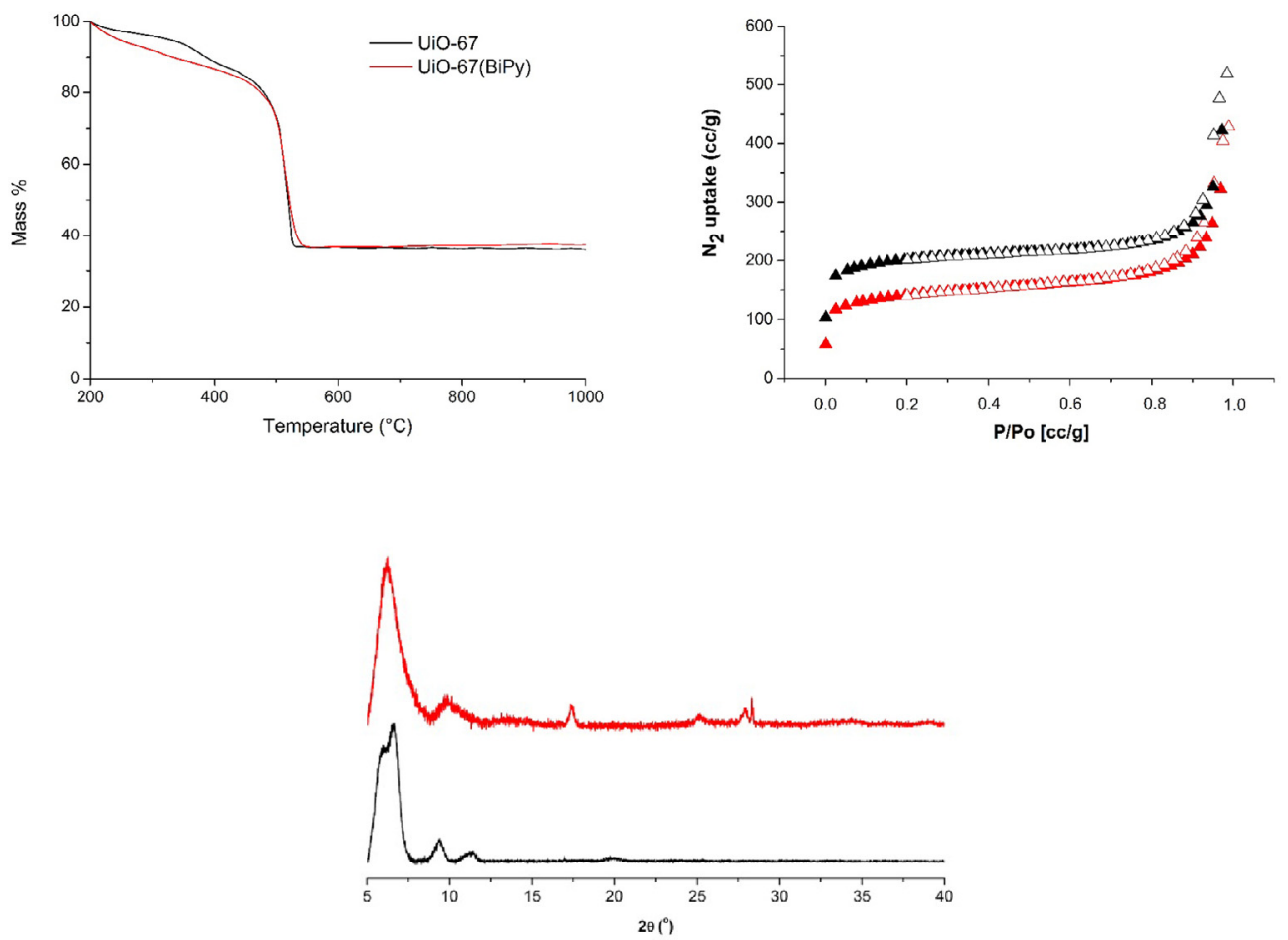

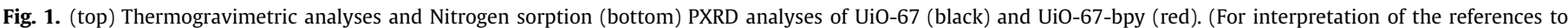
colour in this figure legend, the reader is referred to the web version of this article.) 
MM400 vibratory ball-mill (vbm), in the presence of BPDC in Teflon jars with a $12 \mathrm{~mm}$ diameter zirconium oxide ball, at a frequency of $30 \mathrm{~Hz}$, for $3 \mathrm{~h}$ (Scheme 1).

Reactions were performed under liquid-assisted grinding [19] using a small amount of methanol or DMF $\left(\eta=0.67 \mu \mathrm{L} \cdot \mathrm{mg}^{-1}\right)$ Insoluble materials were recovered after milling, washed with DMF and then $\mathrm{MeOH}$ to remove unreacted substrates. The best results, in terms of purity of the material, were obtained using cluster 1 as substrate and DMF as grinding assistant. A similar protocol was used, starting from cluster 1, for the synthesis of UiO-67bpy, featuring 2,2'-bipyridine-5,5'-dicarboxylic acid as organic ligand. Ui0-67 and UiO-67-bpy were then analysed by nitrogen sorption (BET), thermogravimetric analyses and Powder X-ray diffraction (PXRD) (Fig. 1) and values were compared with those observed for the MOFs obtained by a classical solvothermal route.

Through TGA results, it was possible to determine the thermal stability, the ratio between the organic and the inorganic fractions as well as the purity of the two different MOFs. The results for the two MOFs are quite similar due to the equivalent molecular weight of both ligand and to an equal thermal stability of the MOF. They exhibit a first mass loss before $450{ }^{\circ} \mathrm{C}$ corresponding to the attached solvents (water and DMF molecules) and a total decomposition at $515{ }^{\circ} \mathrm{C}$ of the organic part to form the Zirconium Oxide. The results are consistent with the formation of materials with the UiO phase $\left(\mathrm{Zr}_{6} \mathrm{~L}_{6}(\mathrm{OH})_{4}\left(\mathrm{H}_{2} \mathrm{O}\right)_{4}\right)$ and the formation of $\mathrm{ZrO}_{2}$ (obs. $36.5 \%$, calcd $34.5 \%)$.

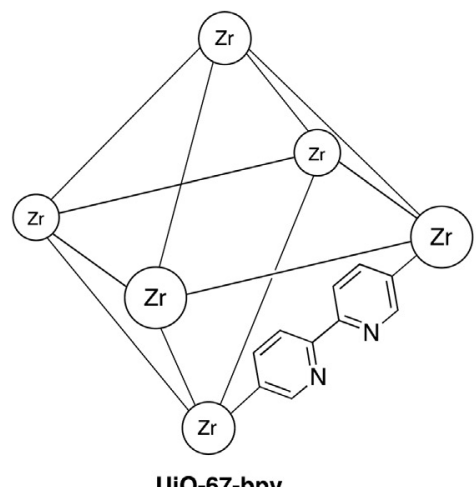

UiO-67-bpy

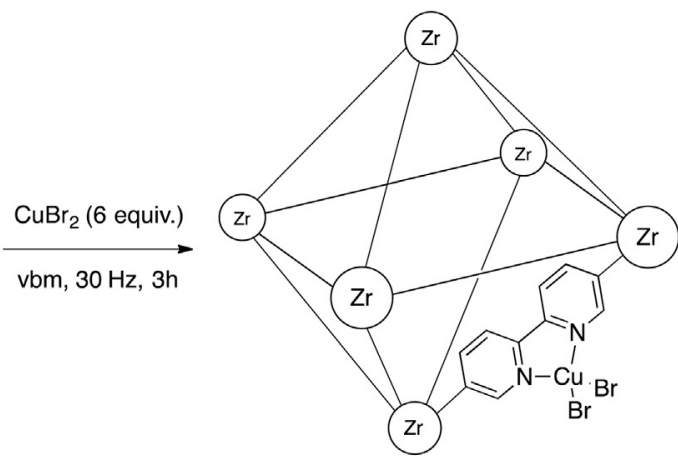

UiO-67-bpy-Cu

Scheme 2. Mechanochemical and post-synthetic modification of UiO-67-bpy.
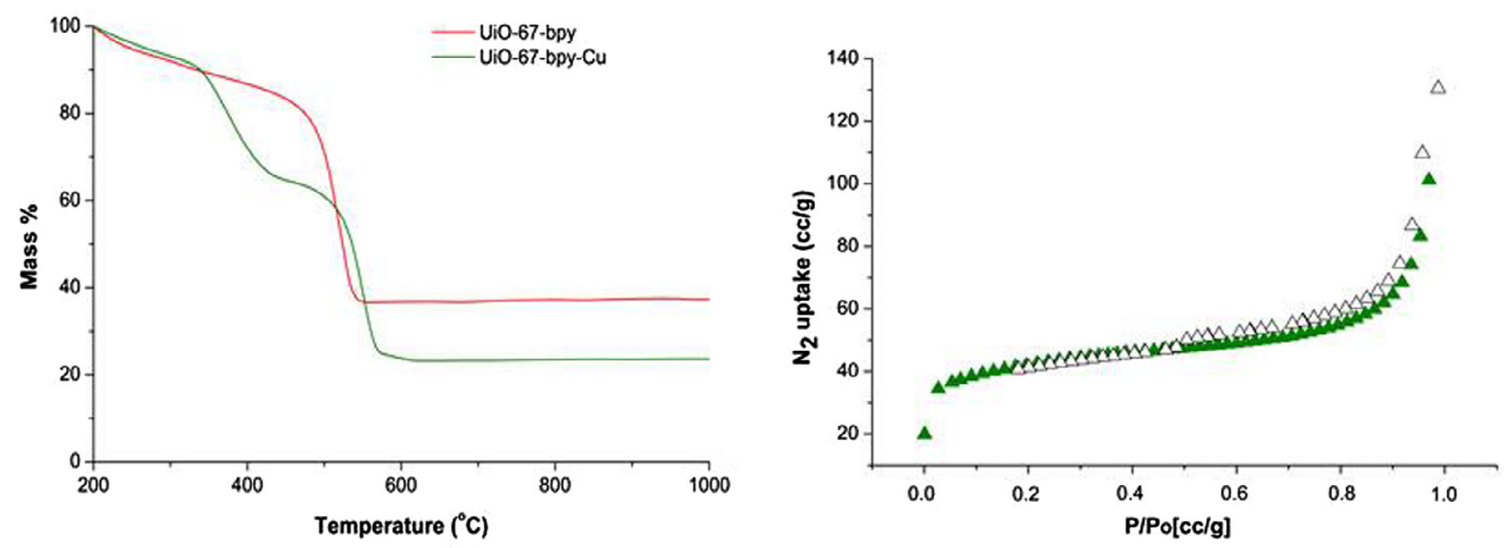

UiO-67-bpy UiO-67-bpy-Cu

Fig. 2. (top) Thermogravimetric analyses of UiO-67-bpy compared to UiO-67-bpy-Cu and Nitrogen sorption of UiO-67-bpy-Cu (bottom) Images of UiO-67-bpy and UiO-67bpy-Cu. 
Permanent porosity of MOFs was determined after the removal of residual solvent molecules under vacuum pump at $100{ }^{\circ} \mathrm{C}$ for $24 \mathrm{~h}$. Nitrogen sorption at $77 \mathrm{~K}$ showed type I isotherms for both materials proving that the MOFs have a microporous structure with a BET surface area of $750 \mathrm{~m}^{2} / \mathrm{g}$ and $650 \mathrm{~m}^{2} / \mathrm{g}$ for Ui0-67 and UiO-67-bpy, respectively. The observed surface areas are lower than those expected for the synthesis of these MOFs by solvothermal syntheses $\left(3000 \mathrm{~m}^{2} / \mathrm{g}\right)$. This is mainly due to the low crystallinity of these materials. PXRD analyses have confirmed the formation and maintenance of the UiO phase. Matching low angle and broad peaks below $15^{\circ}$ indicate small crystalline structure for this UiO. This is due to reaction conditions in the ball-mill that allow the formation of the MOFs but, because of the friction, the crystallinity is not as high as in a solvothermal route [20].

Finally, a post-synthetic modification of Ui0-67-bpy to coordinate copper(II) bromide was realized by solvent-free mechanochemistry (Scheme 2). The formed complex was already demonstrated to oxidize efficiently olefins into epoxides [21]. ICPAES of the digested MOF (in a piranha solution, see SI) has revealed a complete coordination of the bpy ligand sites of the MOF with copper (ratio $\mathrm{L}: \mathrm{Cu}=1: 1$ ) to conduct to a functional MOF with the formula $\mathrm{Zr}_{6} \mathrm{~L}_{6}(\mathrm{OH})_{4}\left(\mathrm{H}_{2} \mathrm{O}\right)_{4}\left(\mathrm{CuBr}_{2}\right)_{6}$. TGA profile of UiO-67-bpy-Cu was quite different of UiO-67-bpy but confirms this formula. In this case, a first mass loss was observed from 200 to $430{ }^{\circ} \mathrm{C}$ corresponding to strongly attached solvent molecules into the framework and a decomposition of the organic moiety at $520^{\circ} \mathrm{C}$ conducting to the formation of the inorganic $\mathrm{ZrO}_{2}$ and $\mathrm{CuO}$ in the expected proportion (obs. 39\%, calcd 35\%). Nitrogen sorption experiment is in accordance with the coordination of the metals and conduct to the reduction of the surface area compared to UiO-67-bpy $\left(155 \mathrm{~m}^{2} / \mathrm{g}\right.$ instead of $650 \mathrm{~m}^{2} / \mathrm{g}$ before coordination). Indeed, incorporation of copper(II) bromide on each bipyridine site of UiO-67-bpy would significantly fill in the pores of the material and thus reduce its ability to adsorb nitrogen Fig. 2.

\section{Conclusion}

We present here for the first time the synthesis of UiO-67 and an iso-structural Ui0-67-bpy by mechanochemistry. The materials were obtained in short reaction time, with a high purity confirmed by TGA analysis. The porosity of the materials was confirmed by nitrogen sorption. We also demonstrated that post-synthetic modification of Ui0-67-bpy was possible under solvent-free ballmilling, giving access to a copper-functionalized material with high purity.

\section{Acknowledgements}

This work was funded by the University Montpellier, CNRS and the French Government through the program "Investissements d'Avenir" ANR-10-LABX-05-01 managed by Agence Nationale pour la Recherche. RNA thanks CONACYT for the scholarship 382043.

\section{References}

[1] C. Sanchez, B. Julián, P. Belleville, M. Popall, Applications of hybrid organicinorganic nanocomposites, J. Mater. Chem. 15 (2005) 3559.

[2] H. Furukawa, K.E. Cordova, M. O'Keeffe, O.M. Yaghi, The chemistry and applications of metal-organic frameworks, Science 341 (2013) 1230444.

[3] P. Horcajada, R. Gref, T. Baati, P.K. Allan, G. Maurin, P. Couvreur, G. Férey, R.E. Morris, C. Serre, Metal-Organic frameworks in biomedicine, Chem. Rev. 112 (2012) 1232-1268.

[4] J.-R. Li, R.J. Kuppler, H.-C. Zhou, Selective gas adsorption and separation in metal-organic frameworks, Chem. Soc. Rev. 38 (2009) 1477.

[5] K. Manna, T. Zhang, M. Carboni, C.W. Abney, W. Lin, Salicylaldimine-based metal-organic framework enabling highly active olefin hydrogenation with iron and cobalt catalysts, J. Am. Chem. Soc. 136 (2014) 13182-13185.

[6] M. Carboni, C.W. Abney, S. Liu, W. Lin, Highly porous and stable metal-organic frameworks for uranium extraction, Chem. Sci. 4 (2013) 2396.

[7] R. Navarro Amador, M. Carboni, D. Meyer, Sorption and photodegradation under visible light irradiation of an organic pollutant by a heterogeneous UiO67-Ru-Ti MOF obtained by post-synthetic exchange, RSC Adv. 7 (2017) 195200.

[8] R. Navarro Amador, M. Carboni, D. Meyer, Photosensitive titanium and zirconium metal organic frameworks: current research and future possibilities, Mater. Lett. 166 (2016) 327-338.

[9] M.J. Katz, Z.J. Brown, Y.J. Colon, P.W. Siu, K.A. Scheidt, R.Q. Snurr, J.T. Hupp, O.K Farha, A facile synthesis of UiO-66, UiO-67 and their derivatives, Chem. Commun. 49 (2013) 9449-9451.

[10] S.L. James, C.J. Adams, C. Bolm, D. Braga, P. Collier, T. Friščić, F. Grepioni, K.D.M Harris, G. Hyett, W. Jones, A. Krebs, J. Mack, L. Maini, A.G. Orpen, I.P. Parkin, W. C. Shearouse, J.W. Steed, D.C. Waddell, Mechanochemistry: opportunities for new and cleaner synthesis, Chem. Soc. Rev. 41 (2012) 413-447.

[11] T. Friščić, Metal-Organic Frameworks: Mechanochemical Synthesis Strategies, in: R.A. Scott (Ed.), Encyclopedia of Inorganic and Bioinorganic Chemistry, John Wiley \& Sons Ltd, Chichester, UK, 2014, pp. 1-19.

[12] K. Užarević, T.C. Wang S.Y. Moon, A.M. Fidelli, J.T. Hupp, O.K. Farha, T. Friščić, Mechanochemical and solvent-free assembly of zirconium-based metalorganic frameworks, Chem. Commun. 52 (2016) 2133-2136.

[13] J.H. Cavka, S. Jakobsen, U. Olsbye, N. Guillou, C. Lamberti, S. Bordiga, K.P. Lillerud, A New Zirconium Inorganic Building Brick Forming Metal Organic Frameworks with Exceptional Stability, J. Am. Chem. Soc. 130 (2008) 13850 13851.

[14] V. Guillerm, S. Gross, C. Serre, T. Devic, M. Bauer, G. Ferey, A zirconium methacrylate oxocluster as precursor for the low-temperature synthesis of porous zirconium(iv) dicarboxylates, Chem. Commun. 46 (2010) 767-769.

[15] A. Beillard, T.-X. Métro, X. Bantreil, J. Martinez, F. Lamaty, Cu(0), O2 and mechanical forces: a saving combination for efficient production of $\mathrm{Cu}-\mathrm{NHC}$ complexes, Chem. Sci. 8 (2017) 1086-1089.

[16] A. Beillard, X. Bantreil, T.-X. Métro, J. Martinez, F. Lamaty, Mechanochemistry for facilitated access to N, N-diaryl NHC metal complexes, New J. Chem. 41 (2017) 1057-1063.

[17] A. Beillard, X. Bantreil, T.-X. Métro, J. Martinez, F. Lamaty, Unraveling the synthesis of homoleptic $[\mathrm{Ag}(\mathrm{N}, \mathrm{N}$-diaryl-NHC $) 2] \mathrm{Y}(\mathrm{Y}=\mathrm{BF} 4, \mathrm{PF} 6)$ complexes by ball-milling, Dalton Trans. 45 (2016) 17859-17866.

[18] A. Beillard, E. Golliard, V. Gillet, X. Bantreil, T.-X. Métro, J. Martinez, F. Lamaty, Expedient Mechanosynthesis of N, N-Dialkyl Imidazoliums and Silver(I)Carbene Complexes in a Ball-Mill, Chem. Eur. J. 21 (2015) 17614-17617.

[19] G.A. Bowmaker, Solvent-assisted mechanochemistry, Chem. Commun. 49 (2013) 334-348.

[20] A. Jayasankar, A. Somwangthanaroj, Z.J. Shao, N. Rodríguez-Hornedo, Cocrystal Formation during Cogrinding and Storage is Mediated by Amorphous Phase, Pharm. Res. 23 (2006) 2381-2392.

[21] T. Toyao, K. Miyahara, M. Fujiwaki, T.-H. Kim, S. Dohshi, Y. Horiuchi, M. Matsuoka, Immobilization of Cu Complex into Zr-Based MOF with Bipyridine Units for Heterogeneous Selective Oxidation, J. Phys. Chem. C 119 (2015) 8131-8137. 